\title{
Review
}

\section{Role of caspase-8 in thymus function}

\author{
N Pozzesi ${ }^{1}$, A Fierabracci ${ }^{2}$, AM Liberati ${ }^{3}$, MP Martelli ${ }^{4}$, E Ayroldi ${ }^{1}$, C Riccardi ${ }^{1}$ and DV Delfino ${ }^{\star, 1}$
}

The thymus is the primary organ responsible for de novo generation of immunocompetent $T$ cells that have a diverse repertoire of antigen recognition. During the developmental process, $98 \%$ of thymocytes die by apoptosis. Thus apoptosis is a dominant process in the thymus and occurs through either death by neglect or negative selection or through induction by stress/aging. Caspase activation is an essential part of the general apoptosis mechanism, and data suggest that caspases may have a role in negative selection; however, it seems more probable that caspase-8 activation is involved in death by neglect, particularly in glucocorticoid-induced thymocyte apoptosis. Caspase-8 is active in double-positive (DP) thymocytes in vivo and can be activated in vitro in DP thymocytes by T-cell receptor (TCR) crosslinking to induce apoptosis. Caspase-8 is a proapoptotic member of the caspase family and is considered an initiator caspase, which is activated upon stimulation of a death receptor (e.g., Fas), recruitment of the adaptor molecule FADD, and recruitment and subsequent processing of procaspase-8. The main role of caspase-8 seems to be pro-apoptotic and, in this review, we will discuss about the involvement of caspase-8 in (1) TCR-triggered thymic apoptosis; (2) death receptor-mediated thymic apoptosis; and (3) glucocorticoid-induced thymic apoptosis. Regarding TCR triggering, caspase-8 is active in medullary, semi-mature heat-stable antigen ${ }^{\text {hi }}\left(H A S^{\text {hi }}\right.$ SP) thymocytes as a consequence of strong TCR stimulation. The death receptors Fas, FADD, and FLIP are involved upstream of caspase-8 activation in apoptosis; whereas, Bid and HDAC7 are involved downstream of caspase-8. Finally, caspase-8 is involved in glucocortocoid-induced thymocyte apoptosis through an activation loop with the protein GILZ. GILZ activates caspase-8, promoting GILZ sumoylation and its protection from proteasomal degradation.

Cell Death and Differentiation (2014) 21, 226-233; doi:10.1038/cdd.2013.166; published online 22 November 2013

\section{Facts}

- Caspase-8 is a proapoptotic member of the cysteine protease family of caspases

- Caspase-8 is activated in the death-inducing signaling complex (DISC), which is induced by death receptor signaling to participate in extrinsic (through direct caspase-3 cleavage) and intrinsic (through Bid cleavage) pathways of apoptosis

- Apoptosis is a fundamental process in thymus physiology as it mediates death by neglect ( $90 \%$ of all thymocytes) and negative selection of autoreactive clones (5\% of all thymocytes) for preventing the development of autoimmune diseases

- The absence of caspase-8 leads to death of mice in utero. Inherited lack of caspase- 8 in humans leads to the development of immunodeficiency disease called caspase-8 deficiency state (CEDS).

\section{Open Questions}

- Is a Fas-independent caspase-8-dependent pathway involved in thymic negative selection or, more generally, in thymic apoptosis?

- Is inhibition of caspase-8 activation important in raising resistance to drug therapy in thymic diseases, such as thymomas?

- Is a better understanding of the relationships between caspase-8, different forms of cFLIP and RIPK1/RIPK3 crucial in driving thymocyte functions towards apoptosis, necroptosis or proliferation?

- What is the biological role of caspase-8-dependent sumoylation/ubiquitination of substrates in thymocyte function?

The thymus is the primary organ responsible for de novo generation of immunocompetent $\mathrm{T}$ cells with a diverse

\footnotetext{
${ }^{1}$ Section of Pharmacology, Toxicology and Chemotherapy, Department of Clinical and Experimental Medicine, University of Perugia, Perugia, Italy; ${ }^{2}$ Research Laboratories, Ospedale Pediatrico Bambino Gesù, Research Institute (IRCCS), Rome, Italy; ${ }^{3}$ Section of Onco-Hematology, Department of Clinical and Experimental Medicine, University of Perugia, Perugia, Italy and ${ }^{4}$ Section of Hematology, Department of Clinical and Experimental Medicine, University of Perugia, Perugia, Italy ${ }^{*}$ Corresponding author: DV Delfino, Section of Pharmacology, Department of Clinical and Experimental Medicine, University of Perugia, Building D, 2nd floor, Piazzale Gambuli, 1, S.Andrea delle Fratte, Perugia 06132, Italy, Tel: +390755858115; Fax: +390755858406; E-mail: domenico.delfino@unipg.it

Keywords: caspase-8; thymus; apoptosis; negative selection; glucocorticoids

Abbreviations: TEC, thymic epithelial cell; DP, double positive; TCR, T-cell receptor; mTEC, medullary TEC; DED, death effector domain; DISC, death-inducing signaling complex; CFLIP, cellular FLICE inhibitory protein; IAP, inhibitor of apoptosis; AIF, apoptosis-inducing factor; SP, single positive; NOD, non-obese diabetic; FAIM, Fas apoptosis inhibitory molecule; HDAC7, histone deacetylase 7; GC, glucocorticoid; GR, GC receptor; DEX, dexamethasone; MPS, methylprednisolone Received 15.7.13; revised 20.8.13; accepted 12.9.13; Edited by J Cidlowski; published online 22.11.2013
} 
repertoire for antigen recognition. However, this organ decreases in size with age in a process termed age-related thymic involution, which is evolutionarily conserved in vertebrates ${ }^{1}$. The thymus is composed of thymocytes of hematopoietic origin and thymic epithelial cells (TECs) of nonhematopoietic origin. The latter comprises the main stromal niche, termed the thymic epithelial space, which is involved in supporting T-cell development and maturation. ${ }^{2}$ T-cell development in the thymus is complex, occurring through a multistep process of proliferation, differentiation, and apoptosis. This process produces a repertoire of $T$ cells capable of colonizing peripheral lymphoid organs to defend the body against invading pathogens ${ }^{3}$ and eliminates autoreactive $T$ cells to avoid autoimmune diseases.

The process of T-cell development from bone marrowderived precursors to fully mature $T$ lymphocytes can take up to 1 week. The thymus of a young adult mouse contains approximately $10^{8}-2 \times 10^{8}$ thymocytes. About $5 \times 10^{7}$ new cells are generated daily; however, only approximately $10^{6}$ to $2 \times 10^{6}(2-4 \%)$ of these cells leave the thymus each day as mature T cells. Despite the disparity between the number of $T$ cells generated and the number exiting, the thymus does not continue to grow in size or cell number. This is because approximately $98 \%$ of thymocytes that develop in the thymus are eliminated by apoptosis. ${ }^{3,4}$ This eradication process reflects the stringent screening that each thymocyte must undergo in order for the body to recognize self-peptide/self$\mathrm{MHC}$ complexes and develop self-tolerance. ${ }^{5}$

Apoptosis in the thymus occurs through death by neglect or negative selection. Stress and aging have also been shown to induce apoptosis in this organ. Immature $\mathrm{CD}^{+}{ }^{+} \mathrm{CD} 8^{+}$ double-positive (DP) thymocytes that fail to generate functional antigen receptors or receive any T-cell receptor (TCR) signals die by neglect, whereas potentially autoreactive thymocytes are eliminated by apoptosis through negative selection $^{6}$ (Figure 1). Death by neglect occurs in DP thymocytes with insufficient affinity to self-antigens; however, the precise mechanism of this process remains unresolved. Negative selection depends on interactions between the (nonself) antigen peptide associated with $\mathrm{MHC}$ molecules on the surface of medullary TECs (mTECs) and the TCR complex on the surface of DP thymocytes. If this binding is strong, DP thymocytes undergo apoptosis and autoreactive thymocyte clones are negatively selected. Thus, the appropriate interaction between mTECs and the TCR complex on DP thymocytes is crucial for distinguishing between positively and negatively selected thymocytes. ${ }^{7,8}$

Two major pathways leading to apoptosis have been described in the thymus, namely the intrinsic and extrinsic pathways. Caspase-8, a member of the caspase family of apoptotic cysteine proteases, is involved in both pathways. Considered an initiator caspase, this protease consists of a relatively large $\mathrm{N}$-terminal dimerization domain and a death effector domain (DED, present in caspase-8 and -10). Like other initiator caspases, caspase- 8 is expressed as a monomer that requires dimerization for activation.

Caspase-8 is activated by the DISC following death receptor signaling. ${ }^{9}$ Upon ligation, death receptors such as Fas multimerize in the cell membrane, leading to a conformational change in the intracellular domain of the receptor. ${ }^{10,11}$

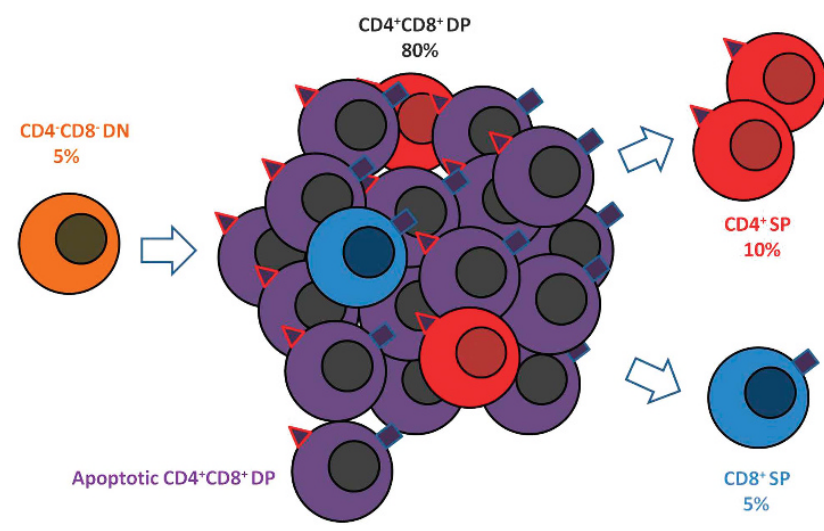

Figure 1 Importance of apoptosis inside the thymus. The development of thymocytes to mature $T$ cells takes place inside thymus. There are four basic steps in this process. (1) A few $\mathrm{CD} 4^{-} \mathrm{CD} 8^{-} \mathrm{DN}$ cells (orange color) originating from the bone marrow proliferate and differentiate into $\mathrm{CD} 4{ }^{+} \mathrm{CD} 8^{+}$DP cells. (2) A large majority $(80 \%)$ of these cells die by neglect $(75 \%)$ or by negative selection $(5 \%)$ (violet cells). Only a small percentage of thymocytes survive by positive selection, and these become (3) $\mathrm{CD} 4^{+} \mathrm{SP}$ (red cells) or (4) $\mathrm{CD} 8^{+} \mathrm{SP}$ (turquoise) cells ready to migrate to peripheral lymphoid organs

This, in turn, leads to recruitment and multimerization of the adaptor protein FADD, which contains a death domain that interacts with the receptor and a DED that recruits monomeric caspase-8. ${ }^{12}$ Caspase- 8 then undergoes dimerization and activation through induced proximity. ${ }^{13,14}$ The subsequent cleavage of caspase-8 stabilizes the dimer and leads to caspase species with increased activity. ${ }^{15-21}$ Finally, caspase-8 cleaves itself between the DEDs and the large subunit, resulting in the release of a stable dimer from the DISC. 22

An alternative fate is the formation of heterodimers with a homolog of caspase-8, cellular FLICE inhibitory protein (cFLIP), that can also be recruited to the DISC. This homolog contains mutations within the catalytic site that can inhibit caspase- 8 activation. This molecule can be subdivided into

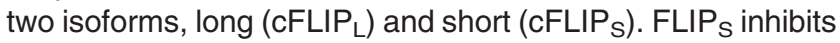
caspase-8 activation, whereas the caspase-8/FLIP $P_{L}$ heterodimer, which contains unprocessed caspase-8, promotes signaling towards cellular survival and activation rather than apoptosis. ${ }^{23}$ Only after the caspase-8 subunit of this heterodimer has been processed does this caspase species become pro-apoptotic. Once activated (i.e., cleaved), caspase- 8 can directly cleave and activate caspase-3 via the extrinsic pathway. Alternatively, activated caspase-8 cleaves Bid, which triggers cytochrome $c$ release from mitochondria that, in turn, determines the activation of caspase- 9 and, ultimately, caspase- 3 via the intrinsic pathway. Thus, caspase- 8 activation is strictly linked to death receptor stimulation. As Bid is a target of caspase-8/10 activity, the intrinsic and extrinsic pathways have been proposed to be interlinked, although probably only in certain cells or tissues. ${ }^{24}$ In the absence of caspase activation, another form of programmed cell death, now generally referred to as 'necroptosis' or 'programmed necrosis', can be triggered. ${ }^{25,26}$ Activation of this pathway is actively opposed by caspases, primarily caspase-8. Active caspase-8 cleaves RIPK1, thereby inhibiting RIPK1dependent necroptosis of the cell. For a detailed overview on 
the general features of caspase-8, we refer the reader to the review by van Raam and Salvesen. ${ }^{27}$

Caspase-8 knockout mice exhibit the most dramatic phenotype of all caspase knockout animals. These mice die in utero around day 11 of gestation with primary defects in cardiac development, growth retardation, and hematopoietic progenitor deficiency. ${ }^{28}$ Importantly, knock down of two other components of the caspase- 8 activation pathway, FADD and FLIP $P_{L}$, results in a similar phenotype. ${ }^{29,30}$ These results indicate that the DISC and caspase- 8 activation are not only critical for triggering apoptosis but also essential for embryonic development. Furthermore, catalytically active caspase-8 is required for rescuing lymphocyte development in caspase8-deficient mice. ${ }^{31}$ In humans, caspase-8 deficiency does not prevent development. Homozygous individuals manifest defective lymphocyte apoptosis and homeostasis and also exhibit defects in the activation of $T$ lymphocytes, $B$ lymphocytes, and natural killer cells, which leads to immunodeficiency. ${ }^{32}$

Although caspase-8 is also involved in survival and development, this protease functions primarily to trigger apoptosis. In this review, we will discuss the role of (1) caspases in thymic apoptosis; (2) caspase-8 in TCR-triggered thymic apoptosis; (3) caspase-8 in death receptor-mediated thymic apoptosis; and (4) caspase-8 in GC-induced thymic apoptosis.

\section{Caspases and Thymic Apoptosis}

Research has established that caspase activation has an essential role in apoptosis. However, the role of caspases in thymic negative selection remains unclear. Although studies have previously reported that peptide-mediated thymocyte deletion can be blocked by the pan-caspase inhibitor zVAD$\mathrm{FMK}^{33}$ more recent reports show that $\mathrm{zVAD}-\mathrm{FMK}$ is ineffective at halting negative selection in TCR transgenic mice. ${ }^{34,35}$ Analysis of transgenic mice expressing p35, a general caspase inhibitor that binds caspase-1, -3, -4, -6, -7, and -8 , has led to mixed results regarding caspase activity in negative selection. ${ }^{36}$ Furthermore, the abrogation of Apaf-1, which is part of the cytochrome c/Apaf-1/caspase-9 complex, does not perturb negative selection. ${ }^{34}$ However, overexpression of inhibitor of apoptosis (IAP), a protein that binds to and inhibits caspase activity, leads to partial inhibition of negative selection. ${ }^{37}$ Taken together, these data suggest that caspases may have a role in negative selection; however, caspase-independent pathways alone have been shown to be sufficient in inducing negative selection. These pathways may include the translocation of apoptosis-inducing factor (AIF) or DNase EndoG from the mitochondria to the nucleus and other unidentified pathways. ${ }^{38,39}$ Nevertheless, it seems more probable that caspase activation is involved in death by neglect and, specifically, in GC-induced thymocyte apoptosis that may be involved in this process. ${ }^{40}$ Finally, only certain caspases, such as caspase-3 and caspase-8-like proteases but not caspase-1, are active in DP thymocytes in vivo and can be activated when DP thymocytes are induced to undergo apoptosis in vitro by TCR crosslinking. ${ }^{41}$

\section{Caspase-8 in TCR-Triggered Thymocyte Apoptosis}

In the thymus, TCR stimulation represents a crucial step for the positive selection of T lymphocytes, negative selection of autoreactive thymocyte clones, and death by neglect. In order for these processes to occur, at least two different parameters must be aligned, the intensity of TCR stimulation and the thymic subpopulation involved. TCR stimulation intensity determines the fate of the stimulated cell. If the intensity is low or absent, thymocytes will die by neglect; however, with moderate stimulation, thymocytes will survive and be positively selected. Strong TCR stimulation promotes thymocyte apoptosis by negative selection.

At least two thymic subpopulations participate in these processes: DP thymocytes (the main subset involved) and medullary semi-mature heat-stable antigen ${ }^{\text {hi }}$ (HSA $\left.{ }^{\text {hi }}\right) \mathrm{CD}^{+}$ $\mathrm{CD}^{-}$single-positive (SP) thymocytes. TCR stimulation of DP thymocytes leads to Fas-independent apoptosis regardless of the strength of TCR stimulation. This mechanism was identified based on studies of Fas-deficient Ipr/lpr cells and non-obese diabetic (NOD) mice. On the other hand, in medullary semi-mature $\mathrm{HSA}^{\text {hi }} \mathrm{CD} 4^{+} \mathrm{CD} 8^{-} \mathrm{SP}$ thymocytes, TCR-induced apoptosis is Fas independent following lowto-intermediate TCR stimulation but is Fas/caspase-8-dependent with strong TCR stimulation. ${ }^{42}$ These findings have also been observed in vivo in peptide-injected TCR-transgenic mice. ${ }^{43}$ Thus, caspase- 8 activation in DP thymocytes ${ }^{41}$ is Fas independent, while caspase-8 activation in medullary semimature $\mathrm{HSA}^{\mathrm{hi}} \mathrm{SP}$ thymocytes is Fas dependent as a consequence of strong TCR stimulation ${ }^{42}$ (Figure 2).

In Fas-deficient Ipr/lpr and NOD mice, the defect in Fas/ caspase-8-dependent apoptosis following strong TCR stimulation is associated with a predisposition towards developing autoimmune diseases. These observations suggest that Fasdependent caspase- 8 activation may be, at least in part, involved in the elimination of autoreactive T-cell clones by negative selection. This defect was most prominent in

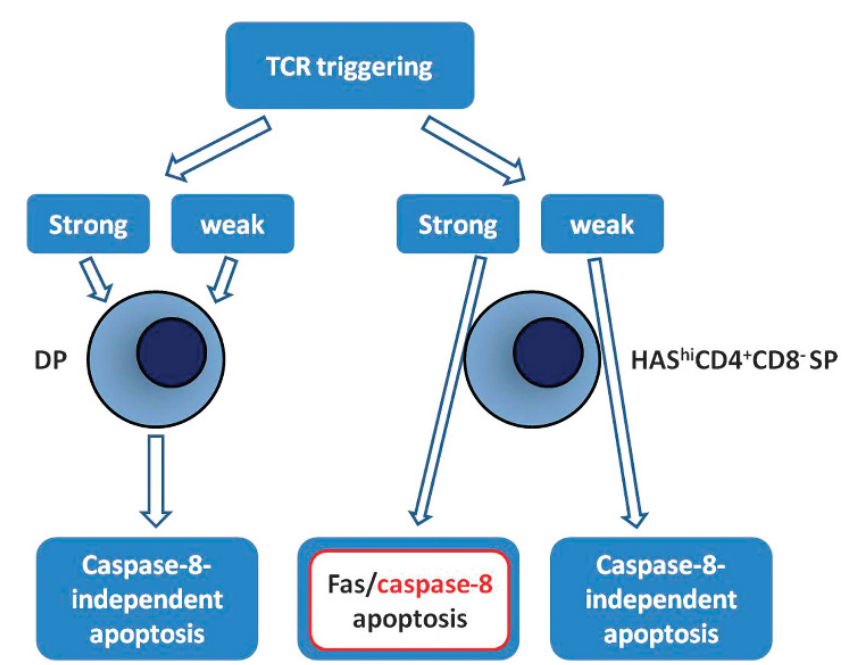

Figure 2 TCR-triggered thymocyte apoptosis. TCR triggering determines apoptosis in two types of thymocyte subpopulations: (1) $\mathrm{CD} 4{ }^{+} \mathrm{CD} 8{ }^{+} \mathrm{DP}$ and (2) $\mathrm{HAS}^{\text {hi }} \mathrm{CD} 4^{+} \mathrm{CD}^{-}$SP cells. Apoptosis is dependent on the Fas/caspase-8 pathway only when strong TCR stimulation occurs in $\mathrm{HAS}^{\text {hi }} \mathrm{CD} 4^{+} \mathrm{CD} 8{ }^{-} \mathrm{SP}$ cells 
medullary semi-mature $\mathrm{HSA}^{\text {hi }} \mathrm{CD} 4{ }^{+} \mathrm{CD} 8{ }^{-}$thymocytes and correlates with the upregulation of cFLIP, the principal inhibitor of caspase- 8 activation in the DISC, ${ }^{42}$ in response to a strong TCR signal. Nevertheless, the role of cFLIP is complex. Despite inhibition of Fas/caspase-8-dependent apoptosis, the number of thymocytes in viral FLIP transgenic mice is lower than in control littermates. Moreover, these thymocytes respond to CD3-mediated stimulation, suggesting that TCR-mediated stimulation may be Fas/caspase-8 independent. ${ }^{44}$ In support of this possibility, cFLIP has been shown to trigger a RIPK1/RIPK3-dependent necroptosis pathway that results in cell death. ${ }^{45,46}$

Another important negative regulator of apoptosis involved in TCR-mediated thymocyte cell death is Fas apoptosis inhibitory molecule (FAIM) ${ }^{47}$. FAIM is upregulated in thymocytes upon TCR engagement and blocks caspase-8 and -9 activity. $\mathrm{FAIM}^{-1-}$ thymocytes are, in fact, highly susceptible to TCR-mediated apoptosis with increased activation of caspase-8 and -9. Furthermore, injection of anti-CD3 antibody leads to augmented depletion of $\mathrm{CD} 4{ }^{+} \mathrm{CD} 8{ }^{+} \mathrm{T}$ cells in the thymus of $\mathrm{FAIM}^{-1-}$ mice compared with wild-type controls, suggesting that FAIM has a role in inhibiting thymocyte apoptosis. ${ }^{48}$ TCR crosslinking of $\mathrm{FAIM}^{-1-}$ thymocytes leads to elevated levels of the orphan nuclear receptor Nur77, which has a role in thymocyte apoptosis. Interestingly, in the absence of FAIM, Nur77 ubiquitination and degradation is reduced. AAIM $^{-1-}$ thymocytes also exhibit a defect in TCRinduced activation of Akt, whose activity is required for Nur77 ubiquitination. Further analyses utilizing FAIM-deficient primary thymocytes and FAIM-overexpressing DO-11.10 T cells indicate that FAIM acts upstream of Akt, thereby promoting its localization to lipid rafts and affecting its activation. Taken together, these studies define a TCR-induced FAIM/Akt/ Nur77 signaling axis that is critical for modulating the apoptosis of developing thymocytes. ${ }^{48}$ One intriguing hypothesis proposes that FAIM may be involved when TCR triggering shifts thymocyte fate towards survival (positive selection) instead of death (negative selection).

\section{Involvement of Caspase-8 in Death Receptor-Stimulated Thymocyte Apoptosis}

Indirect information about the involvement of caspase-8 activation in death receptor-dependent thymocyte apoptosis was gleaned from studies in which molecules upstream or downstream of caspase-8 were inhibited or knocked down (Figure 3).

\section{Upstream molecules}

Fas: The work of Kishimoto and Sprent ${ }^{42}$ indicates that the Fas pathway is involved in negative selection but only in a small population of semi-mature SP thymocytes. However, the Fas pathway is active in DP thymocytes, indicating its involvement in processes other than negative selection and thus, possibly, in death by neglect. Of all the death receptor pathways, Fas signaling has a critical role in thymocyte apoptosis; therefore, Fas receptor expression must be strictly regulated in thymocytes. As mentioned previously, FAIM is a negative regulator of Fas. ${ }^{48}$ One positive regulator of Fas is MSSP, a transcription factor that regulates c-myc, alpha-



Figure 3 Molecules involved in the caspase-8 pathway in the thymus. Caspase-8 is activated through stimulation of Fas and recruitment of FADD. Upstream molecules that negatively regulate this pathway are FAIM and FLIP, whereas MSSP positively regulates the Fas/caspase-8 pathway. Once activated, caspase-8 cleaves and activates downstream caspase-3, HDAC7, and Bid, thus promoting thymocyte apoptosis

smooth actin, MHC class I, MHC class 2, and thyrotrophin receptor. Thymocytes from MSSP knockout mice exhibit reduced Fas expression, resulting in abrogation of Fas/ caspase-8-mediated induction of apoptosis. ${ }^{49}$ Moreover, lipid raft disruption by cholesterol depletion abolishes Fas-triggered recruitment of FADD and caspase-8 to the membrane, DISC formation, and cell death. ${ }^{50}$

FADD: As mentioned above, upon triggering, Fas recruits FADD which, in turn, recruits caspase-8 to form the DISC. FADD is critical for viability as FADD-deficient homozygous mice die in utero. In newborn chimeras, thymocytes from $F A D D^{-/-}$animals are completely resistant to Fas-mediated apoptosis but less sensitive to TNF-mediated apoptosis, indicating a lack of redundancy in Fas-mediated apoptotic pathways. However, thymocyte subpopulations were apparently normal, as they resembled those of wild-type mice, while the thymocyte number decreased to an undetectable level with age. These results along with the observed similarities between $\mathrm{FADD}^{-/-}$and IL-2R beta-deficient mice suggest that: (1) Fas/FADD-dependent caspase-8 activation is not crucial for thymocyte development and (2) that there is an unexpected association between cell proliferation and apoptosis. ${ }^{51}$ The latter could be due to caspase-8-mediated proliferative effects. ${ }^{27}$ Data from FADD-deficient mice published by Winoto and colleagues ${ }^{51}$ were recently confirmed in another model. T-cell-specific FADD-deficient mice were shown to possess normal numbers of thymocytes and slightly reduced peripheral $T$ cell numbers, whereas B-cellspecific FADD deletion led to increased peripheral B-cell numbers. ${ }^{52}$

cFLIP: cFLIP can be recruited by FADD in the DISC instead of or in association with caspase-8. Although CFLIPS $_{S}$ is composed solely of two DEDs, in addition to these domains, cFLIP $\mathrm{L}_{\mathrm{L}}$ contains an (inactive) caspase-like domain that renders the protein structurally similar to pro-caspase-8. Both CFLIP $_{S}$ and $\mathrm{CFLIP}_{\mathrm{L}}$ suppress apoptosis by inhibiting caspase-8 activation, albeit at different levels of 
pro-caspase- 8 processing. To investigate the consequences of deregulated $\mathrm{CFLIP}_{\mathrm{S}}$ expression in vivo, Ick/cFLIP $\mathrm{S}^{-}$ transgenic mice overexpressing CFLIP $_{S}$ in thymocytes and mature $T$ cells were established. As expected, Fas ligandinduced apoptosis was impaired in these transgenic $\mathrm{T}$ cells. However, thymic and splenic cell numbers, as well as CD4/ CD8 cellularity, were normal in these animals. Unlike in Fasdeficient mice, Thy ${ }^{+} \mathrm{B} 220^{+} \mathrm{CD}^{-}{ }^{-} \mathrm{CD} 8^{-}$peripheral $\mathrm{T}$ cells did not accumulate in the $/ c k / c F L I P_{S}$-transgenic mice. Despite the capacity of cFLIPS $_{S}$ to inhibit Fas-induced apoptosis, T-cell lymphomagenesis was not observed in


$\mathrm{V} \beta 8^{+}$memory $\mathrm{T}$ cells increased upon staphylococcal enterotoxin $B$ injection, suggesting a specific in vivo function for cFLIPS $_{S}$ in the maintenance of restimulated $T$ cells. ${ }^{53}$ Finally, the total number of thymocytes was reduced in 4-8week-old FLIP-overexpressing transgenic mice in which thymocytes were resistant to Fas-induced apoptosis, further suggesting that Fas-dependent caspase-8 activation and apoptosis is not involved in thymocyte development. Notably, this reduction was also observed in FLIP transgenic mice with a $\mathrm{Fas}^{-1-}$ background, suggesting that this reduction is Fas independent. ${ }^{44}$

TRAIL: The importance of TNF family of death receptors, other than Fas, in thymocyte negative selection has been the subject of many studies, with equivocal results. Thus, in order to clarify the role of the FADD/caspase- 8 pathway in this process, intrathymic negative selection of TRAILdeficient thymocytes has been investigated using four well-established models, including antibody-mediated $\mathrm{TCR} / \mathrm{CD} 3$ ligation in vitro, stimulation with endogenous superantigen in vitro and in vivo, and treatment with exogenous superantigen in vitro. These models were unable to demonstrate a role for TRAIL signaling, suggesting that this pathway is not a critical factor for thymocyte negative selection. ${ }^{54}$

IRF5: The transcription factor IFN regulatory factor 5 (IRF5), which is involved in the activation of innate immune responses, has recently been shown to be critical for DNA damage-induced apoptosis and tumor suppression. In particular, IRF5 is involved in a stage of Fas signaling that precedes the activation of caspase- 8 and c-Jun N-terminal kinase. In addition to hepatocytes, IRF5 is also required for apoptosis in dendritic cells (DCs) activated by hypomethylated CpG but not in thymocytes and embryonic fibroblasts in vitro. Thus, these findings reveal a cell type-specific function for IRF5 in the complex regulatory mechanism of death receptor-induced apoptosis. ${ }^{55}$

\section{Downstream molecules}

Bid: Studies using Bid-deficient mice demonstrated that this proapoptotic Bcl-2 family member is not involved in thymocyte development. When $\mathrm{Bid}^{-1-}$ thymocytes were treated in vitro with anti-Fas antibody, caspase-8 remained active, but the caspase-8-dependent mitochondrial apoptotic pathway was blocked. In these thymocytes, mitochondrial dysfunction was delayed, cytochrome $c$ was not released, effector caspase activity was reduced, and cleavage of apoptotic substrates was altered. Thus the Fas/caspase-8mediated mitochondrial apoptotic pathway is important in thymocytes, ${ }^{56}$ but this pathway does not appear to be critical for thymocyte development.

Histone deacetylase 7 (HDAC7): Several novel putative caspase-8 substrates were identified by the prediction of protease specificity program. One such protein was HDAC7, which was shown to undergo cleavage faster than any other caspase-8 substrate described to date. Studies have demonstrated that HDAC7 is cleaved in primary $\mathrm{CD}^{+}$ $\mathrm{CD}^{+}$thymocytes undergoing extrinsic apoptosis. Importantly, cleavage of HDAC7 alters its subcellular localization and abrogates its ability to repress Nur77. Thus, these data demonstrate a direct role for initiator caspase-mediated proteolysis in promoting gene transcription. ${ }^{57}$ In immature $\mathrm{CD}^{+}{ }^{+} \mathrm{CD}^{+}{ }^{+}$thymocytes, HDAC7 represses, in fact, the transcription of Nur77, a pro-apoptotic protein involved in the death of $\mathrm{T}$ lymphocytes during negative selection. ${ }^{58,59} \mathrm{~T}$-cell receptor stimulation of immature thymocytes triggers nuclear export of HDAC7. This leads to a loss of transcription repression and a MEF2D-dependent induction of Nur77 expression and apoptosis. ${ }^{60,61}$

Two possible outcomes of caspase-8-mediated HDAC7 cleavage in thymocytes can be envisaged. First, cleavage could promote expression of Nur77 and feed forward to enhance apoptosis. This may be important for promoting apoptosis in cells that have reduced levels to executioner caspases or high levels of apoptosis inhibitors, such as XIAP. Alternatively, if the N-terminal cleavage fragment is transported back into the nucleus, a dominant-negative effect may result. The caspase-generated $\mathrm{N}$-terminal fragment of HDAC7 may bind to MEF2D, preventing transcription of Nur77 in a way that cannot be switched off, because it cannot be exported to the nucleus, promoting thymocyte cell survival. This hypothesis is challenged by the recognized instability of the caspase-generated HDAC7 N-terminal fragment. ${ }^{62}$

\section{Role of Caspase-8 in GC-Induced Apoptosis in Thymocytes}

Although initiation of GC-induced thymic apoptosis relies on the $\mathrm{GC}$ receptor (GR) and de novo gene expression, the effector phase differs among cell types. Proteasomal degradation, as well as caspase-3, -8 , and -9 activity, is essential for GC-induced apoptosis in murine thymocytes; however, these enzymes are dispensable in splenic T cells. The role of GCinduced apoptosis, including the involvement of caspase-8, in the thymus is still controversial. (Please refer to the excellent review by Ashwell et al. for more information about this field $^{63}$ ). Although some studies demonstrated that caspase-8 activation is dispensable for thymocyte apoptosis, others have shown it to be critical. Mann et al. ${ }^{64}$ showed that both spontaneous and GC-induced thymocyte apoptosis are associated with both cellular depolarization and repression of $\mathrm{Na}^{+} / \mathrm{K}^{+}$-ATPase. These effects were blocked by the pancaspase inhibitor (zVAD) but not by specific inhibitors of caspase-8, -9, and -3. Among GCs, methylprednisolone (MPS) induces early stages of rat thymocyte apoptosis, as indicated by an increase in Annexin-V-positive events and a sequential increase in NO production by mitochondrial and endoplasmic reticulum membranes. MPS activates caspase6 and caspase-3 but not caspase-8. Finally, the thymocyte 
apoptotic pathway induced by MPS primarily involves the mitochondria but not the endoplasmic reticulum. ${ }^{65}$

Other studies have focused on the critical role of caspase-8 in GC-induced thymocyte apoptosis. In one model of in vitro thymocyte apoptosis, caspase-8 inhibition abrogated cytochrome $c$ release, caspase- 9 and caspase- 3 activation, and apoptosis, suggesting that caspase- 8 inhibition can indirectly inhibit caspase-9 and/or that dexamethasone (DEX)-induced caspase- 8 activation is upstream of mitochondria. Thus, it can regulate caspase-3 directly or through cytochrome $c$ release and, ultimately, caspase-9/caspase-3 activation. Critical signaling events upstream of caspase-8 activation include expression of Src kinase, PI-PLC, and aSMase as inhibition of their synthesis abolished caspase-8 processing and apoptosis. ${ }^{66}$ Another study showed that caspase-8 activation may be critical for promoting GC-induced thymocyte apoptosis. More specifically, mouse thymocyte apoptosis was triggered by DEX-induced caspase-8, -3 , and -9 processing along with caspase-8-independent loss of mitochondrial membrane potential. This DEX-induced apoptosis was found to be dependent on caspase-8 but not caspase-9, as it was abolished by inhibition of caspase- 8 and -3 but not caspase- 9 processing. Inhibition of sphingosine production reduced caspase- 8 activation. In addition, inhibition of the proteasome impaired activation of all three caspases. Altogether, these data indicate that DEX-induced apoptosis is mediated, in part, by sphingosine that, along with proteasome activity, contributes to the processing of caspase- 8 and caspase- 3 independent of mitochondria. ${ }^{67}$

Besides promoting thymocyte apoptosis, GCs modulate the transcription of numerous genes that have an important role in triggering thymic apoptosis. For example, GC-induced leucine zipper (GILZ) is strongly upregulated in the thymus and has been demonstrated to function in thymocyte development. GILZ was originally identified in 1997, during a systematic study of genes transcriptionally induced by GCs and responsible for GC-activated apoptosis. ${ }^{68}$ Subsequently, GILZ was shown to mediate several GC functions, such as modulation of T-lymphocyte activation, IL-2 production, apoptosis, and cell proliferation. ${ }^{69-71}$ As the basic characteristics and regulation of the GILZ protein were being clarified, many unexpected features of this protein began to emerge, which provided new insight into the physiological role and function of GILZ itself. Because of the variety of protein interactions and its abundance in several cell types, GILZ has, in fact, a crucial role in controlling protein trafficking and signaling. Most recently, novel functions have been defined, including regulation of T helper cell differentiation ${ }^{72,73}$ and DC function, ${ }^{74,75}$ increase of epithelial $\mathrm{Na}^{+}$channel-mediated $\mathrm{Na}^{+}$transport in the kidney, ${ }^{76,77}$ and control of malignant transformation through inhibition of Ras-driven tumorigenesis. ${ }^{71,78,79}$ Young adult GILZ-overexpressing transgenic mice exhibit dramatically reduced numbers of $\mathrm{CD} 4^{+} \mathrm{CD} 8^{+}$ thymocytes and increased ex vivo thymocyte apoptosis. Apoptotic pathway analysis detected reduced antiapoptotic $\mathrm{Bcl}-\mathrm{xL}$ expression and increased activation of caspase- 8 and caspase-3 in a sequential manner. In older GILZ-transgenic mice, perturbation of thymic subset numbers was amplified over time, as demonstrated by further reduction in $\mathrm{CD} 4^{+}$ $\mathrm{CD}^{+}$cells and increase in $\mathrm{CD} 4^{+} \mathrm{CD} 8^{-}, \mathrm{CD} 4^{-} \mathrm{CD} 8^{-}$, and
$\mathrm{CD}^{+}{ }^{+} \mathrm{CD}^{-}{ }^{-}$cell numbers. Thus, GILZ appears to be part of a novel pro-apoptotic GC pathway. ${ }^{70}$ Possible cross-talk between GILZ and caspase-8 in DEX-treated thymocytes has been evaluated. The expression of DEX-induced GILZ protein was reduced when caspase-8 activity was inhibited. Inhibition of the proteasome abrogated this reduction in GILZ expression, suggesting that DEX-induced caspase-8 activation protects GILZ from degradation. It has been hypothesized that this caspase-8-dependent protection of GILZ could be due to caspase-8-driven sumoylation, which consequently inhibits GILZ ubiquitination. In fact, GILZ binding to SUMO-1 in vitro and in vivo is dependent on caspase- 8 activation. This correlates with studies, reported in the same article, on caspase-8-deficient mice and cell lines, which display a concomitant reduction in GILZ expression in thymocytes and lymphocytes. ${ }^{80}$ These results suggest that caspase- 8 activation protects GILZ from proteasomal degradation and induces its binding to SUMO-1 in GC-treated thymocytes. ${ }^{80}$ Taken together, these results suggest a feedback loop between GILZ and caspase- 8 that begins with GR-mediated Gilz gene expression. Once expressed, GILZ induces caspase-8 activation, which in turn promotes GILZ maintenance through its sumoylation and subsequent inhibition of its ubiquitination/ proteasomal degradation. Thus, GC-dependent transcription of Gilz is necessary but not sufficient for its expression. In order to promote its expression, GCs must also protect GILZ from proteasomal degradation through caspase-8 activation (Figure 4).

Regarding death receptors involvement by GCs, the expression pattern of Fas and DR5, both inducing cell death through caspase-8, was analyzed in the thymus after either $\gamma$-irradiation or DEX treatment. Both Fas and DR5 were induced in the thymus by ionizing radiation in a p53dependent manner, whereas only DR5 was induced by DEX in a p53-independent manner. ${ }^{81}$ Studies have established that caspase- 8 is activated during GC-induced thymic apoptosis. Nevertheless, the importance of this activation in the economy of the apoptotic process in the thymus remains controversial. The discrepancy observed in the different



Figure 4 The GILZ-caspase-8 loop. The binding of GCs to their GR stimulates transcription of Gilz. Once Gilz is translated, it activates caspase-8 from procaspase-8, and the active caspase-8 causes Sumo-1 to bind GILZ. Sumo-1 binding competes with ubiquitin binding and inhibits proteasomal degradation of GILZ 
studies could be due to the various experimental conditions tested, such as the type of GC used, the strain of mice, and the different apoptotic pathways stimulated by GCs. Different GCs have, in fact, different apoptotic activity that is related to their ability to induce both genomic (e.g., caspase-8, -9, and -3 activation and GILZ expression) and non-genomic effects (e.g., PI-PLC phosphorylation). ${ }^{82}$ For instance, live imaging by confocal microscopy revealed that lysosomal cathepsin B, an unrecognized component of this pathway to date, becomes rapidly activated in thymocytes after GC exposure. This is followed by leakage of cathepsin B into the cytosol, nuclear condensation, and processing of caspase-8 and -3 . Collectively, the apoptotic program induced by GCs is comprised of cell type-specific and shared features. ${ }^{83}$

The biological significance of GC-induced thymocyte apoptosis remains unclear. It is reasonable to think that GCs can participate in death by neglect and age- and stressrelated thymic involution. A new interesting finding suggests that GC-induced caspase-8-dependent thymocyte apoptosis is involved in thymic atrophy as a consequence of acute Trypanosoma cruzi infection. Blockade of GR activity with RU486 prevented DP thymocyte apoptosis together with caspase- 8 activation in a murine model of acute $T$. cruzi infection. These findings indicate that DP T-cell apoptosis following experimental $T$. cruzi infection is dependent on GC stimulation for promoting caspase- 8 activation. ${ }^{84}$

\section{Conclusions}

Caspase- 8 is a critical molecule as its absence leads to death of mice in utero. Upon activation, its main function is to promote apoptosis and, in thymus, apoptosis of negative selection is critical to eliminate autoaggressive T-cell clones that, if not eliminated, could contribute to develop autoimmune diseases. Despite the involvement of caspase-8 in apoptosis, there is no clear evidence demonstrating the involvement of caspase- 8 in impairing thymic central tolerance. Thus, the role of caspase- 8 in the thymus is still uncertain, although this is probably the consequence of an inability to experimentally dissect this issue. As an example, all data on caspase-8 in the thymus were gathered by decreasing its expression. However, it would be very informative if data on caspase-8 overexpression were also available. An alternative hypothesis is that the role of caspase-8 in thymic apoptosis is not critical, whereas caspase- 8 would rather be involved in thymocyte proliferation. This hypothesis is supported by studies on CEDS, a rare human disease in which the main clinical manifestation is immunodeficiency, and this implies an involvement of caspase-8 in thymocyte proliferation rather than in apoptosis. ${ }^{85}$ This review highlights the role of caspase8 in TCR-triggered, death receptor-triggered, and GC-induced apoptosis. Questions remain as to whether or not the thymus is involved in autoimmune symptoms present in humans with caspase-8-deficiency and if this could be a consequence of impaired negative selection. Additionally, it is important to further elucidate the novel function of caspase-8 in inhibiting proteasomal degradation of other molecules involved in apoptosis and proliferation besides GILZ. Future studies will clarify these questions and potentially enable the development of therapeutic strategies that modulate caspase-8 activation for the treatment of immunodeficiency state or other unknown caspase-8-dependent disorders.

\section{Conflict of Interest}

The authors declare no conflict of interest.

1. Shanley DP, Aw D, Manley NR, Palmer DB. An evolutionary perspective on the mechanisms of immunosenescence. Trends immunol 2009; 30: 374-381.

2. Gui J, Mustachio LM, Su DM, Craig RW. Thymus size and age-related thymic involution: early programming, sexual dimorphism, progenitors and stroma. Aging Dis 2012; 3: 280-290.

3. Shortman K, Egerton M, Spangrude GJ, Scollay R. The generation and fate of thymocytes. Semin Immunol 1990; 2: 3-12.

4. Surh CD, Sprent J. T-cell apoptosis detected in situ during positive and negative selection in the thymus. Nature 1994; 372: 100-103.

5. Janaway Jr CA, Travers P, Walport M, Shlomchik MJ. The development and survival of lymphocytes. In: Immunobiology, the immune system in health and disease. Garland Science Publishing: New York, 2005, pp 251-257.

6. Godfrey DI, Purton JF, Boyd RL, Cole TJ. Stress-free T-cell development: glucocorticoids are not obligatory. Immunol Today 2000; 21: 606-611.

7. Alam SM, Davies GM, Lin CM, Zal T, Nasholds W, Jameson SC et al. Qualitative and quantitative differences in $\mathrm{T}$ cell receptor binding of agonist and antagonist ligands. Immunity 1999; 10: 227-237.

8. Gronski MA, Boulter JM, Moskophidis D, Nguyen LT, Holmberg K, Elford AR et al. TCR affinity and negative regulation limit autoimmunity. Nat Med 2004; 10: 1234-1239.

9. Mace PD, Riedl SJ. Molecular cell death platforms and assemblies. Curr Opin Cell Biol 2010; 22: 828-836.

10. Scott FL, Stec B, Pop C, Dobaczewska MK, Lee JJ, Monosov E et al. The Fas-FADD death domain complex structure unravels signalling by receptor clustering. Nature 2009; 457 : 1019-1022.

11. Wang L, Yang JK, Kabaleeswaran V, Rice AJ, Cruz AC, Park AY et al. The Fas-FADD death domain complex structure reveals the basis of DISC assembly and disease mutations. Nat Struct Mol Biol 2010; 17: 1324-1329.

12. Tu S, McStay GP, Boucher LM, Mak T, Beere HM, Green DR. In situ trapping of activated initiator caspases reveals a role for caspase-2 in heat shock-induced apoptosis. Nature Cell Biol 2006; 8: 72-77.

13. Boatright KM, Renatus M, Scott FL, Sperandio S, Shin H, Pedersen IM et al. A unified model for apical caspase activation. Mol Cell 2003; 11: 529-541.

14. Muzio M, Stockwell BR, Stennicke HR, Salvesen GS, Dixit VM. An induced proximity model for caspase-8 activation. J Biol Chem 1998; 273: 2926-2930.

15. Keller N, Mares J, Zerbe O, Grutter MG. Structural and biochemical studies on procaspase8: new insights on initiator caspase activation. Structure 2009; 17: 438-448.

16. Keller N, Grutter MG, Zerbe O. Studies of the molecular mechanism of caspase-8 activation by solution NMR. Cell Death Differ 2010; 17: 710-718.

17. Oberst A, Pop C, Tremblay AG, Blais V, Denault JB, Salvesen GS et al. Inducible dimerization and inducible cleavage reveal a requirement for both processes in caspase-8 activation. J Biol Chem 2010; 285: 16632-16642.

18. Pop C, Fitzgerald P, Green DR, Salvesen GS. Role of proteolysis in caspase- 8 activation and stabilization. Biochemistry 2007; 46: 4398-4407.

19. Pop C, Oberst A, Drag M, Van Raam BJ, Riedl SJ, Green DR et al. FLIP(L) induces caspase 8 activity in the absence of interdomain caspase 8 cleavage and alters substrate specificity. Biochem J 2011; 433: 447-457.

20. Chang DW, Xing Z, Capacio VL, Peter ME, Yang X. Interdimer processing mechanism of procaspase-8 activation. EMBO J 2003; 22: 4132-4142.

21. Donepudi M, Mac Sweeney A, Briand C, Grutter MG. Insights into the regulatory mechanism for caspase-8 activation. Mol Cell 2003; 11: 543-549.

22. Medema JP, Scaffidi C, Kischkel FC, Shevchenko A, Mann M, Krammer PH et al. FLICE is activated by association with the CD95 death-inducing signaling complex (DISC). EMBO J 1997; 16: 2794-2804.

23. Hughes MA, Harper N, Butterworth M, Cain K, Cohen GM, MacFarlane M. Reconstitution of the death-inducing signaling complex reveals a substrate switch that determines CD95-mediated death or survival. Mol Cell 2009; 35: 265-279.

24. Kantari $\mathrm{C}$, Walczak H. Caspase-8 and bid: caught in the act between death receptors and mitochondria. Biochim Biophys Acta 2011; 1813: 558-563.

25. Degterev A, Hitomi J, Germscheid M, Ch'en IL, Korkina O, Teng X et al. Identification of RIP1 kinase as a specific cellular target of necrostatins. Nat Chem Biol 2008; 4: 313-321.

26. Declercq W, Vanden Berghe T, Vandenabeele P. RIP kinases at the crossroads of cell death and survival. Cell 2009; 138: 229-232.

27. van Raam BJ, Salvesen GS. Proliferative versus apoptotic functions of caspase-8 Hetero or homo: the caspase-8 dimer controls cell fate. Biochim Biophys Acta 2012; 1824: 113-122.

28. Varfolomeev EE, Schuchmann M, Luria V, Chiannilkulchai N, Beckmann JS, Mett IL et al. Targeted disruption of the mouse Caspase 8 gene ablates cell death induction by the TNF receptors, Fas/Apo1, and DR3 and is lethal prenatally. Immunity 1998; 9: 267-276. 
29. Yeh WC, Pompa JL, McCurrach ME, Shu HB, Elia AJ, Shahinian A et al. FADD: essentia for embryo development and signaling from some, but not all, inducers of apoptosis. Science 1998; 279: 1954-1958.

30. Yeh WC, Itie A, Elia AJ, Ng M, Shu HB, Wakeham A et al. Requirement for Casper (c-FLIP) in regulation of death receptor-induced apoptosis and embryonic development. Immunity 2000; 12: 633-642.

31. Leverrier S, Salvesen GS, Walsh CM. Enzymatically active single chain caspase-8 maintains T-cell survival during clonal expansion. Cell Death Differ 2011; 18: 90-98.

32. Chun HJ, Zheng L, Ahmad M, Wang J, Speirs CK, Siegel RM et al. Pleiotropic defects in lymphocyte activation caused by caspase-8 mutations lead to human immunodeficiency. Nature 2002; 419: 395-399.

33. Clayton LK, Ghendler Y, Mizoguchi E, Patch RJ, Ocain TD, Orth K et al. T-cell receptor ligation by peptide/MHC induces activation of a caspase in immature thymocytes: the molecular basis of negative selection. EMBO J 1997; 16: 2282-2293.

34. Hara H, Takeda A, Takeuchi M, Wakeham AC, Itie A, Sasaki M et al. The apoptotic protease-activating factor 1-mediated pathway of apoptosis is dispensable for negative selection of thymocytes. J Immunol 2002; 168: 2288-2295.

35. Doerfler P, Forbush KA, Perlmutter RM. Caspase enzyme activity is not essential for apoptosis during thymocyte development. J Immunol 2000; 164: 4071-4079.

36. Izquierdo M, Grandien A, Criado LM, Robles S, Leonardo E, Albar JP et al. Blocked negative selection of developing T cells in mice expressing the baculovirus p35 caspase inhibitor. EMBO J 1999; 18: 156-166.

37. Robles MS, Leonardo E, Criado LM, Izquierdo M, Martinez AC. Inhibitor of apoptosis protein from Orgyia pseudotsugata nuclear polyhedrosis virus provides a costimulatory signal required for optimal proliferation of developing thymocytes. J Immunol 2002; 168: 1770-1779.

38. Joza N, Susin SA, Daugas E, Stanford WL, Cho SK, Li CY et al. Essential role of the mitochondrial apoptosis-inducing factor in programmed cell death. Nature 2001; 410: 549-554.

39. Li LY, Luo $X$, Wang $X$. Endonuclease $G$ is an apoptotic DNase when released from mitochondria. Nature 2001; 412: 95-99.

40. Delfino DV, Pozzesi N, Pierangeli S, Ayroldi E, Fierabracci A. Manipulating thymic apoptosis for future therapy of autoimmune diseases. Curr Pharm Des 2011; 17: 3108-3119.

41. Jiang $D$, Zheng L, Lenardo MJ. Caspases in T-cell receptor-induced thymocyte apoptosis Cell Death Differ 1999; 6: 402-411.

42. Kishimoto H, Sprent J. A defect in central tolerance in NOD mice. Nat Immunol 2001; 2 1025-1031.

43. Kishimoto $\mathrm{H}$, Surh $\mathrm{CD}$, Sprent J. A role for Fas in negative selection of thymocytes in vivo. $J$ Exp Med 1998; 187: 1427-1438.

44. OhYama T, Tsukumo S, Yajima N, Sakamaki K, Yonehara S. Reduction of thymocyte numbers in transgenic mice expressing viral FLICE-inhibitory protein in a Fas-independent manner. Microbiol Immunol 2000; 44: 289-297.

45. Oberst A, Dillon CP, Weinlich R, McCormick LL, Fitzgerald P, Pop C et al. Catalytic activity of the caspase-8-FLIP(L) complex inhibits RIPK3-dependent necrosis. Nature 2011; 471 363-367.

46. Kaiser WJ, Upton JW, Long AB, Livingston-Rosanoff D, Daley-Bauer LP, Hakem R et al. RIP3 mediates the embryonic lethality of caspase-8-deficient mice. Nature 2011; 471 368-372.

47. Zhong X, Schneider TJ, Cabral DS, Donohoe TJ, Rothstein TL. An alternatively spliced long form of Fas apoptosis inhibitory molecule (FAIM) with tissue-specific expression in the brain. Mol Immunol 2001; 38: 65-72.

48. Huo J, Xu S, Lam KP. Fas apoptosis inhibitory molecule regulates $T$ cell receptor-mediated apoptosis of thymocytes by modulating Akt activation and Nur77 expression. J Biol Chem 2010; 285: 11827-11835.

49. Nomura J, Matsumoto K, Iguchi-Ariga SM, Ariga H. Mitochondria-independent induction of Fas-mediated apoptosis by MSSP. Oncol Rep 2005; 14: 1305-1309.

50. Hueber AO, Bernard AM, Herincs Z, Couzinet A, He HT. An essential role for membrane rafts in the initiation of Fas/CD95-triggered cell death in mouse thymocytes. EMBO Rep 2002; 3: 190-196.

51. Zhang J, Cado D, Chen A, Kabra NH, Winoto A. Fas-mediated apoptosis and activationinduced T-cell proliferation are defective in mice lacking FADD/Mort1. Nature 1998; 392 : 296-300.

52. Rosenberg S, Zhang H, Zhang J. FADD deficiency impairs early hematopoiesis in the bone marrow. J Immunol 2011; 186: 203-213.

53. Oehme I, Neumann F, Bosser S, Zornig M. Transgenic overexpression of the Caspase-8 inhibitor FLIP(short) leads to impaired T cell proliferation and an increased memory T cell pool after staphylococcal enterotoxin B injection. Eur J Immunol 2005; 35: 1240-1249.

54. Cretney E, Uldrich AP, Berzins SP, Strasser A, Godfrey DI, Smyth MJ. Normal thymocyte negative selection in TRAlL-deficient mice. J Exp Med 2003; 198: 491-496.

55. Couzinet A, Tamura K, Chen HM, Nishimura K, Wang Z, Morishita $Y$ et al. A cell-typespecific requirement for IFN regulatory factor 5 (IRF5) in Fas-induced apoptosis. Proc Nat Acad Sci USA 2008; 105: 2556-2561.

56. Yin XM, Wang K, Gross A, Zhao Y, Zinkel S, Klocke B et al. Bid-deficient mice are resistant to Fas-induced hepatocellular apoptosis. Nature 1999; 400: 886-891.

57. Scott FL, Fuchs GJ, Boyd SE, Denault JB, Hawkins CJ, Dequiedt F et al. Caspase-8 cleaves histone deacetylase 7 and abolishes its transcription repressor function J Biol Chem 2008; 283: 19499-19510.

58. He YW. Orphan nuclear receptors in T lymphocyte development. J Leuk Biol 2002; 72 440-446.
59. Siggs OM, Makaroff LE, Liston A. The why and how of thymocyte negative selection. Curr Opin Immunol 2006; 18: 175-183.

60. Dequiedt F, Kasler H, Fischle W, Kiermer V, Weinstein M, Herndier BG et al. HDAC7, a thymus-specific class II histone deacetylase, regulates Nur77 transcription and TCR-mediated apoptosis. Immunity 2003; 18: 687-698.

61. Parra M, Kasler H, McKinsey TA, Olson EN, Verdin E. Protein kinase D1 phosphorylates HDAC7 and induces its nuclear export after T-cell receptor activation. J Biol Chem 2005; 280: 13762-13770.

62. Li X, Song S, Liu Y, Ko SH, Kao HY. Phosphorylation of the histone deacetylase 7 modulates its stability and association with 14-3-3 proteins. J Biol Chem 2004; 279 : 34201-34208

63. Ashwell JD, Lu FW, Vacchio MS. Glucocorticoids in T cell development and function*. Annu Rev Immunol 2000; 18: 309-345.

64. Mann CL, Bortner CD, Jewell CM, Cidlowski JA. Glucocorticoid-induced plasma membrane depolarization during thymocyte apoptosis: association with cell shrinkage and degradation of the $\mathrm{Na}(+) / \mathrm{K}(+)$-adenosine triphosphatase. Endocrinology 2001; 142: 5059-5068.

65. Bustamante J, Bersier G, Badin RA, Cymeryng C, Parodi A, Boveris A. Sequential NO production by mitochondria and endoplasmic reticulum during induced apoptosis. Nitric Oxide 2002; 6: 333-341.

66. Marchetti MC, Di Marco B, Cifone G, Migliorati G, Riccardi C. Dexamethasone-induced apoptosis of thymocytes: role of glucocorticoid receptor-associated Src kinase and caspase-8 activation. Blood 2003; 101: 585-593.

67. Lepine S, Lakatos B, Courageot MP, Le Stunff H, Sulpice JC, Giraud F. Sphingosine contributes to glucocorticoid-induced apoptosis of thymocytes independently of the mitochondrial pathway. J Immunol 2004; 173: 3783-3790.

68. D'Adamio F, Zollo O, Moraca R, Ayroldi E, Bruscoli S, Bartoli A et al. A new dexamethasone-induced gene of the leucine zipper family protects $T$ lymphocytes from TCR/CD3-activated cell death. Immunity 1997; 7: 803-812.

69. Ayroldi E, Migliorati G, Bruscoli S, Marchetti C, Zollo O, Cannarile L et al. Modulation of T-cell activation by the glucocorticoid-induced leucine zipper factor via inhibition of nuclear factor kappaB. Blood 2001; 98: 743-753.

70. Delfino DV, Agostini M, Spinicelli S, Vito P, Riccardi C. Decrease of Bcl-xL and augmentation of thymocyte apoptosis in GILZ overexpressing transgenic mice. Blood 2004: 104: 4134-4141.

71. Ayroldi E, Zollo O, Bastianelli A, Marchetti C, Agostini M, Di Virgilio R et al. GILZ mediates the antiproliferative activity of glucocorticoids by negative regulation of Ras signaling. J Clin Invest 2007; 117: 1605-1615.

72. Cannarile L, Fallarino F, Agostini M, Cuzzocrea S, Mazzon E, Vacca C et al. Increased GILZ expression in transgenic mice up-regulates Th-2 lymphokines. Blood 2006; 107: 1039-1047.

73. Cannarile L, Cuzzocrea S, Santucci L, Agostini M, Mazzon E, Esposito E et al. Glucocorticoid-induced leucine zipper is protective in Th1-mediated models of colitis. Gastroenterology 2009; 136: 530-541.

74. Cohen N, Mouly E, Hamdi H, Maillot MC, Pallardy M, Godot V et al. GILZ expression in human dendritic cells redirects their maturation and prevents antigen-specific $T$ lymphocyte response. Blood 2006; 107: 2037-2044.

75. Hamdi H, Godot V, Maillot MC, Prejean MV, Cohen N, Krzysiek R et al. Induction of antigen-specific regulatory $\mathrm{T}$ lymphocytes by human dendritic cells expressing the glucocorticoid-induced leucine zipper. Blood 2007; 110: 211-219.

76. Muller OG, Parnova RG, Centeno G, Rossier BC, Firsov D, Horisberger JD. Mineralocorticoid effects in the kidney: correlation between alphaENaC, GILZ, and Sgk-1 mRNA expression and urinary excretion of $\mathrm{Na}+$ and K +. J Am Soc Nephrol 2003; 14: 1107-1115.

77. Soundararajan R, Zhang TT, Wang J, Vandewalle A, Pearce D. A novel role for glucocorticoid-induced leucine zipper protein in epithelial sodium channel-mediated sodium transport. J Biol Chem 2005; 280: 39970-39981.

78. Ayroldi E, Riccardi C. Glucocorticoid-induced leucine zipper (GILZ): a new important mediator of glucocorticoid action. FASEB J 2009; 23: 3649-3658.

79. Ayroldi E, Cannarile L, Migliorati G, Nocentini G, Delfino DV, Riccardi C. Mechanisms of the anti-inflammatory effects of glucocorticoids: genomic and nongenomic interference with MAPK signaling pathways. FASEB J 2012; 26: 4805-4820.

80. Delfino DV, Spinicelli S, Pozzesi N, Pierangeli S, Velardi E, Bruscoli S et al. Glucocorticoidinduced activation of caspase-8 protects the glucocorticoid-induced protein Gilz from proteasomal degradation and induces its binding to SUMO-1 in murine thymocytes. Cell Death Differ 2011; 18: 183-190.

81. Burns TF, Bernhard EJ, El-Deiry WS. Tissue specific expression of p53 target genes suggests a key role for KILLER/DR5 in p53-dependent apoptosis in vivo. Oncogene 2001; 20: 4601-4612.

82. Bruscoli S, Di Virgilio R, Donato V, Velardi E, Baldoni M, Marchetti C et al. Genomic and non-genomic effects of different glucocorticoids on mouse thymocyte apoptosis. Eur J Pharmacol 2006; 529: 63-70.

83. Wang D, Muller N, McPherson KG, Reichardt HM. Glucocorticoids engage different signal transduction pathways to induce apoptosis in thymocytes and mature T cells. J Immunol 2006; 176: 1695-1702.

84. Farias-de-Oliveira DA, Villa-Verde DM, Nunes Panzenhagen PH, Silva dos Santos D, Berbert LR, Savino W et al. Caspase-8 and caspase-9 mediate thymocyte apoptosis in Trypanosoma cruzi acutely infected mice. J Leuk Biol 2013; 93: 227-234.

85. Bidere N, Su HC, Lenardo MJ. Genetic disorders of programmed cell death in the immune system. Annu Rev Immunol 2006; 24: 321-352. 